\section{Anticoncepcionais orais e pressão arterial - pesquisa epidemiológica de hipertensão arterial no Rio Grande do Sul}

- Escola Nacional de Satide

Puiblica - FIOCRUZ -

Rio de Janeiro

* Secretaria de Estado de Saúde e Higiene do Rio de Janeiro e Escola Nacional de Saúde Pública - FIOCRUZ - Rio de Janeiro

The present paper describes the relationship between the use of oral contraceptives and arterial blood pressure among women residing in rural, urban and metropolitan areas of Rio Grande do Sul, the southermost state of Brazil. The analysis is based on data from a cross-sectional survey of blood pressure carried out in Rio Grande do Sul, in 1978. In an analysis of covariance, controlling for age and Quetelet Index, the average systolic and diastolic pressures of oral contraceptive users or ex-users were compared to the average pressure of never-users. A positive difference in systolic pressure was observed systematically for all four study strata (Rural Interior, Urban Interior, Capital and its Metropolitan Belt), among women using oral contraceptives at the time of interview. The difference was found to be significant ( $p<1 \%$ ) for women residing in the Metropolitan Belt using oral contraceptives for more than 2 years. The effect on diastolic pressure, although generally positive, were smaller and not significant.

The research methodology allowed for the association between the use of oral contraceptives and other risk factors to be evaluated. The effect on blood pressure was found to be most intense for women of the lowest educational status residing in the Metropolitan belt. For the group of women with a history of hypertension during pregnancy or classified as hypertensive (criteria 140/90) the effect of oral contraceptives was also found to be highly significant $(p<1 \%)$ - an alteration of over $10 \mathrm{~mm} \mathrm{Hg}$ on the average systolic pressure. These results suggest that the use of oral contraceptives brings about a greater increase in systolic pressure in groups of women with normally higher levels of pressure.

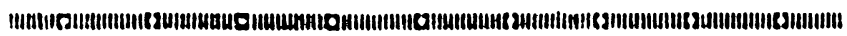

\title{
INTRODUÇÃO
}

O Brasil tem mostrado um declínio acentuado de fecun- 
didade na última década, com uma queda de aproximadamente $25 \%$ no período de 1970 a $1976^{1}$. Pesquisadores têm demonstrado que a anticoncepção, em suas diferentes formas, desempenha um papel de importância na redução da fecundidade ${ }^{5}$.

Investigaçōes realizadas no Brasil, entre 1979 e 1981 , indicam uma maior proporção de mulheres fazendo uso de anticoncepcionais nas Regiões Sul e Sudeste. Diferenças interregionais foram também encontradas para os tipos de métodos utilizados: enquanto no Norte e em alguns Estados nordestinos a prevalência de esterilização feminina alcança níveis superiores que a proporção de mulheres que fazem uso da pílula, no Sul do país o comportamento é inverso ${ }^{12}$.

Vários pesquisadores têm estudado a relação entre o uso de anticoncepcionais orais e níveis tensionais nos países de. senvolvidos $2,4,6,10,11,14,15$. Porém, resultados de estudos semelhantes em países com dinâmica populacional e condições sócio-econômicas como as do Brasil não são disponiveis.

O presente trabalho, a partir dos dados da Pesquisa de Hipertensão Arterial no Rio Grande do $\mathrm{Sul}^{3}$, se propõe a descrever a relação entre o uso de anticoncepcionais orais e a pressão arterial em mulheres residentes nas áreas urbana, rural e metropolitana nesse Estado. Além disso, propõe-se a analisar também outros fatores de risco que, conjunta. mente com o uso de anticoncepcionais orais, poderiam causar alterações na pressão arterial.

O Rio Grande do Sul é o Estado do Brasil que apresenta maior uso de métodos contraceptivos em geral. Entre as mulheres no periodo reprodutivo, $40 \%$ fazem uso da pílu$\mathrm{la}^{8}$. Desta maneira, este estudo contribui para avaliar o impacto do uso de anticoncepcionais orais numa população onde pouco se conhece sobre o uso de anticoncepcionais, nos vários grupos sócio-econômicos, como também para analisar a associação do consumo com os níveis tensionais.

\section{METODOLOGIA}

Neste estudo foram consideradas cerca de 1.300 mulheres, de cor branca, na idade de 20-49 anos, que fizeram parte do inquérito da Pesquisa Epidemiológica de Hipertensão Arterial no Rio Grande do $\mathrm{Sul}^{3}$.

A amostra desta pesquisa foi selecionada no Estado do Rio Grande do Sul, subdividido em quatro estratos geoeconômicos, assim descritos: Porto Alegre, a capital do Estado - Cinturão Metropolitano, constituído pelos municípios periféricos em torno de Porto Alegre onde se concentra o setor industrial, - Interior Urbano, formado por todos os 
outros municípios urbanos, - Interior Rural, composto por todas as áreas não definidas como urbanas.

O plano de amostragem da pesquisa foi descrito anteriormente ${ }^{3}$

Para todos os indivíduos participantes do inquérito, foram feitas duas medidas de pressão sistólica e diastólica, na posição sentada, tendo sido utilizado um aparelho Tycos modificado, o ENSP-esfigmomanômetro ${ }^{3}$. Os examinadores foram especialmente treinados pelo método de Rose ${ }^{7}$.

Para as mulheres casadas ou que tinham pelo menos um filho, perguntas foram feitas sobre o uso de pilulas anticoncepcionais e. em caso de resposta afirmativa, sobre o tempo de uso do medicamento.

Para essas mulheres, tínhamos informações adicionais sobre idade, peso, altura, cor e nível de instrução, assim como relativas a hábitos de fumar e beber. Para mulheres que já tinham tido uma gestação, perguntou-se também se elas apresentaram algum problema na gravidez, como hipertensão, inchaço, falta de ar ou convulsão.

O tipo de amostragem da pesquisa nos permitiu avaliar, comparativamente, a utilização de pílulas anticoncepcionais por área - urbana, rural ou metropolitana. No entanto. o número pequeno de mulheres pretas não nos permitiu estratificar por cor.

A análise estatística consistiu em estimar prevalências do uso de anticoncepcionais orais em cada um dos quatro estratos geoeconômicos, pesquisar associações do uso de pílulas com hipertensão em mulheres (critério 140/90), assim como efeitos do uso nas pressões arteriais médias.

Para pesquisar efeitos do uso de anticoncepcionais orais nas médias de pressões, as mulheres brancas, casadas ou com pelo menos um filho, com idade inferior a 45 anos, foram subdivididas em cinco grupos:

i) as que nunca tomaram pílulas;

ii) as que tomam atualmente, por tempo inferior a dois anos;

iii) as que tomam atualmente, por tempo superior a dois anos;

iv) as que tomaram por tempo inferior a dois anos, mas não usam atualmente;

v) as que tomaram por tempo superior a dois anos, mas não usam atualmente.

Para estimar diferenças de médias na pressão arterial, foi considerado o seguinte modelo linear:

$$
\mathrm{Y}_{\mathrm{ij}}=\mu+\alpha \mathrm{x}_{\mathrm{ij}}+\beta \mathrm{z}_{\mathrm{ij}}+\gamma_{\mathrm{i}}+\mathrm{e}_{\mathrm{ij}} \quad \mathrm{i}=1,2,3,4,5
$$

sendo a variável critério y, ora a pressão sistólica ( 2 a medi. da), ora a pressão diastólica ( 2 ? medida), controlando-se 
por idade (x) e índice de Quetelet (z)(peso/(altura) ${ }^{2}$ x 1000). No modelo, $o$ indice $i$ representa cada um dos cinco grupos descritos acima, $n_{\mathrm{i}}$ o tamanho de cada grupo e $j$ cada uma das observações dentro de cada grupo.

A comparação das médias na pressão arterial nas mulhe. res que fazem uso de anticoncepcionais atualmente, ou que deixaram de tomar, com aquelas que nunca fizeram uso, foi feita através de contrastes da forma:

$\xi_{j}=\gamma_{j}-\gamma_{1}, i=2,3,4,5$

e seus respectivos testes de significância.

Para as mulheres que residem na área metropolitana (Porto Alegre e Cinturão Metropolitano), foram avaliados, ainda, com o mesmo tipo de análise estatística, efeitos conjuntos do uso de anticoncepcionais orais com hábitos de fumar, obesidade e alterações na gravidez.

\section{RESULTADOS}

$\mathrm{Na}$ Tabela I, apresentamos as proporçōes de mulheres que fazem uso de anticoncepcionais orais, categorizadas por faixa etária, em cada um dos estratos geoeconômicos do Estado do Rio Grande do Sul. Na área metropolitana, somente $32 \%$ das mulheres nunca tomaram pílulas, enquanto que, no interior urbano e no rural, essa proporção aumenta para $46 \%$ e $65 \%$, respectivamente. Nota-se também a divu]. gação cada vez maior do uso de anticoncepcionais orais: em todos os estratos, a proporção das mulheres que nunca tomaram pílula é maior nas faixas etárias mais altas.

\section{TABELA I}

Distribuição de mulheres brancas por uso de pilulas, por faixa etária, por estrato geoeconómico

\begin{tabular}{|c|c|c|c|c|c|c|}
\hline \multirow{2}{*}{$\begin{array}{l}\begin{array}{l}\text { Uso de } \\
\text { Pilula }\end{array} \\
\text { Faixa } \\
\text { E tária }\end{array}$} & \multicolumn{3}{|c|}{ Porto Alegre } & \multicolumn{3}{|c|}{ Cinturão Me tropolitano } \\
\hline & Atualmente & $\begin{array}{c}\text { Nunca } \\
\text { Tomaram }\end{array}$ & $\begin{array}{c}\text { No } \\
\text { Passado }\end{array}$ & Atualmente & $\begin{array}{c}\text { Nunca } \\
\text { Tomaram }\end{array}$ & $\begin{array}{c}\text { No } \\
\text { Passado }\end{array}$ \\
\hline $\begin{array}{c}20-29 \\
\%\end{array}$ & $\begin{array}{l}43 \\
44\end{array}$ & $\begin{array}{l}14 \\
14\end{array}$ & $\begin{array}{l}41 \\
42\end{array}$ & $\begin{array}{l}62 \\
59\end{array}$ & $\begin{array}{l}13 \\
12\end{array}$ & $\begin{array}{l}31 \\
29\end{array}$ \\
\hline $\begin{array}{c}30-39 \\
\%\end{array}$ & $\begin{array}{l}44 \\
41\end{array}$ & $\begin{array}{l}21 \\
20\end{array}$ & $\begin{array}{l}42 \\
39\end{array}$ & $\begin{array}{l}52 \\
41\end{array}$ & $\begin{array}{l}31 \\
24\end{array}$ & $\begin{array}{l}44 \\
35\end{array}$ \\
\hline $\begin{array}{c}40-49 \\
\%\end{array}$ & $\begin{array}{l}12 \\
10 \\
\end{array}$ & $\begin{array}{l}62 \\
52 \\
\end{array}$ & $\begin{array}{l}45 \\
38 \\
\end{array}$ & $\begin{array}{l}18 \\
17 \\
\end{array}$ & $\begin{array}{l}69 \\
63 \\
\end{array}$ & $\begin{array}{l}22 \\
20 \\
\end{array}$ \\
\hline $\begin{array}{c}\text { Total } \\
\%\end{array}$ & $\begin{array}{l}99 \\
31\end{array}$ & $\begin{array}{l}97 \\
30\end{array}$ & $\begin{array}{r}128 \\
39\end{array}$ & $\begin{array}{r}132 \\
39\end{array}$ & $\begin{array}{r}113 \\
33\end{array}$ & $\begin{array}{l}97 \\
28\end{array}$ \\
\hline
\end{tabular}


Para analisar a relação entre o uso de anticoncepcionais e o nivel de instrução, as mulheres foram subdivididas naquelas que são analfabetas ou sabem ler e escrever, mas não têm primário completo - nível 1 de instrução - e naquelas que têm escolaridade igual ou superior ao primário completo nível 2 de instrução. Verificamos que o comportamento é semelhante em todas as faixas etárias, a proporção de mu. lheres que nunca tomaram pílulas é sempre maior para as de menor escolaridade, sendo mais significativo esse resultado nas faixas etárias mais velhas (ver Tabela II). Comparativamente, para cada faixa etária, obtemos $33 \%$ contra $22 \%$, $41 \%$ contra $31 \%$ e $73 \%$ contra $61 \%$ para esta proporção.

É interessante observar que o nivel de instrução das mu. lheres brancas tem evoluido no Rio Grande do Sul. Na faixa etária mais jovem, $22 \%$ das mulheres apresentam escolaridade mais baixa, enquanto que, comparando-se com as outras faixas, encontramos respectivamente $36 \%$ e $54 \%$. Simultaneamente, com o crescimento da escolaridade e da divulgação da pílula, decresce a proporção de mulheres que nunca fizeram uso da mesma (66\%, 34\% e $24 \%)$.

Para avaliar a associação entre hipertensão e o uso de pílulas anticoncepcionais, foram consideradas todas as mulheres brancas, subdivididas apenas pelas três faixas etárias (Tabela III).

Observa-se que, apesar do tamanho pequeno do grupo de hipertensas na faixa etária mais jovem, de 11 mulheres hi. pertensas, 10 tomam pilula atualmente. Nas outras duas classes de idade, o uso de anticoncepcionais não mostrou um aumento significativo na proporção de mulheres hipertensas.

\begin{tabular}{|c|c|c|c|c|c|}
\hline \multicolumn{3}{|c|}{ Interior Urbano } & \multicolumn{3}{|c|}{ Interior Rural } \\
\hline A tualmente & $\begin{array}{c}\text { Nunca } \\
\text { Tomaram }\end{array}$ & $\begin{array}{c}\text { No } \\
\text { Passado }\end{array}$ & Atualmente & $\begin{array}{c}\text { Nunca } \\
\text { Tomaram }\end{array}$ & $\begin{array}{c}\text { No } \\
\text { Passado }\end{array}$ \\
\hline $\begin{array}{l}58 \\
56\end{array}$ & $\begin{array}{l}29 \\
28\end{array}$ & $\begin{array}{l}17 \\
16\end{array}$ & $\begin{array}{l}29 \\
41\end{array}$ & $\begin{array}{l}35 \\
50\end{array}$ & $\begin{array}{l}6 \\
9\end{array}$ \\
\hline $\begin{array}{l}50 \\
42\end{array}$ & $\begin{array}{l}47 \\
39\end{array}$ & $\begin{array}{l}23 \\
19\end{array}$ & $\begin{array}{l}25 \\
24\end{array}$ & $\begin{array}{l}58 \\
57\end{array}$ & $\begin{array}{l}19 \\
19\end{array}$ \\
\hline $\begin{array}{l}20 \\
18 \\
\end{array}$ & $\begin{array}{l}77 \\
70 \\
\end{array}$ & $\begin{array}{l}13 \\
12 \\
\end{array}$ & $\begin{array}{l}12 \\
11 \\
\end{array}$ & $\begin{array}{l}89 \\
82 \\
\end{array}$ & $\begin{array}{l}8 \\
7 \\
\end{array}$ \\
\hline $\begin{array}{r}128 \\
38\end{array}$ & $\begin{array}{r}153 \\
46\end{array}$ & $\begin{array}{l}53 \\
16\end{array}$ & $\begin{array}{l}66 \\
23\end{array}$ & $\begin{array}{r}182 \\
65\end{array}$ & $\begin{array}{l}33 \\
12\end{array}$ \\
\hline
\end{tabular}


TABELA II

Distribuiçäo das mulheres brancas por uso de anticoncepcionais, nivel de instruçāo e faixa etária (quatro estratos conjuntamente)

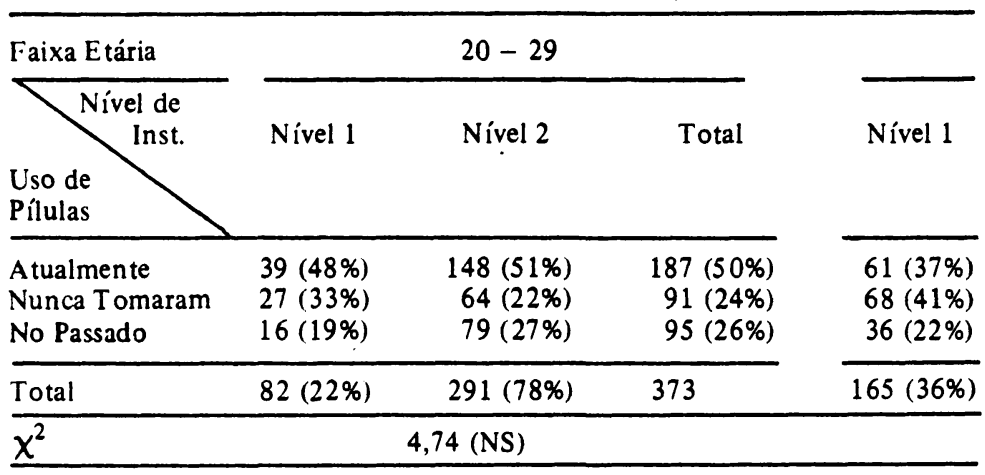

\section{TABELA III}

Associaçāo entre hipertensão (140/90) e uso de pilulas, por faixa etária (quatro estratos conjuntamente)

\begin{tabular}{lcccc}
\hline \multirow{2}{*}{ Condição } & & \multicolumn{3}{c}{$20-29$} \\
\cline { 3 - 5 } & & Atualmente & Nunca & $\begin{array}{c}\text { No } \\
\text { Passado }\end{array}$ \\
\cline { 3 - 5 } Hipertenso & No & 10 & 1 & 0 \\
& $\%$ & 90,9 & 9,1 & 0,0 \\
\multirow{2}{*}{ Normal } & No & 178 & 91 & 95 \\
& $\%$ & 48,9 & 25 & 26,1 \\
\multirow{2}{*}{ Total } & No & 188 & 92 & 95 \\
& $\%$ & 50,1 & 24,5 & 25,3 \\
\hline$\chi^{2}$ & & & 7,75 & \\
\hline
\end{tabular}

$\mathrm{Na}$ Tabela IV, encontram-se as médias de pressão arterial sistólica e diastólica por cada um dos cinco grupos definidos acima e por estrato geoeconômico. Essas médias, no entanto, não estão padronizadas por idade e índice de Quetelet, e, claramente, $o$ grupo das que nunca tomaram pílulas abrange uma proporção maior de mulheres mais velhas.

Através de uma análise de covariâncias, controlando-se idade e índice de Quetelet, as médias de pressão sistólica e diastólica dos quatro grupos, formados pelas usuárias e exusuárias de pílulas, foram comparadas com as médias de pressão das mulheres que nunca tomaram anticoncepcionais. Estimativas dos contrastes, seus desvios padrão e significância, encontram-se nas Tabelas V e VI. 


\begin{tabular}{|c|c|c|c|c|}
\hline \multicolumn{2}{|l|}{$30-39$} & \multicolumn{3}{|c|}{$40-49$} \\
\hline Nível 2 & Total & Nível 1 & Nível 2 & Total \\
\hline $\begin{array}{r}110(38 \%) \\
89(31 \%) \\
92(31 \%)\end{array}$ & $\begin{array}{l}171(38 \%) \\
157(34 \%) \\
128(28 \%)\end{array}$ & $\begin{array}{r}32(16 \%) \\
150(73 \%) \\
23(11 \%)\end{array}$ & $\begin{array}{r}30(12 \%) \\
147(61 \%) \\
65(27 \%)\end{array}$ & $\begin{array}{r}62(14 \%) \\
297(66 \%) \\
88(20 \%)\end{array}$ \\
\hline $192(64 \%)$ & 456 & 205 (46\%) & $242(54 \%)$ & 447 \\
\hline \multicolumn{2}{|c|}{$7.074(\mathrm{p}<5 \%)$} & \multicolumn{3}{|c|}{$17,19(\mathrm{p}<1 \%)$} \\
\hline
\end{tabular}

\begin{tabular}{cccccccc}
\hline & $30-39$ & & & $30-49$ \\
\cline { 5 - 7 } Atualmente & Nunca & $\begin{array}{c}\text { No } \\
\text { Passado }\end{array}$ & & Atualmente & Nunca & $\begin{array}{c}\text { No } \\
\text { Passado }\end{array}$ \\
\cline { 6 - 8 } & & 10 & & 21 & 90 & 28 \\
46 & 18 & & & 15,1 & 64,7 & 20,1 \\
174 & 136 & 120 & & 40 & 208 & 61 \\
36,5 & 33,7 & 29,8 & & 12,9 & 67,3 & 19,7 \\
171 & 154 & 130 & & 61 & 298 & 89 \\
37,6 & 33,8 & 28,6 & & 13,6 & 66,5 & 19,9 \\
\hline
\end{tabular}

Observamos que, para a pressão sistólica, houve sistematicamente, em todos os estratos, uma diferença positiva para as mulheres que estão tomando pílula atualmente, mas esta diferença só se mostrou significativa para as mulheres do Cinturão Metropolitano que tomam pílula há mais de dois anos. Para as mulheres que deixaram de tomar anticoncepcionais, esta diferença mostrou-se sempre negativa, apesar de não-significativa. Para a pressão diastólica, observamos efeitos quase nulos, em geral positivos naquelas que tomam pílula atualmente e negativos nas ex-usuárias, mas não significativos em todos os estratos.

Mulheres residentes na área metropolitana (Porto Alegre ou Cinturão Metropolitano) foram ainda subdivididas por 
TABELA IV

Médias de pressão arterial sistólica e diastólica por uso de pilulas, por estrato geoeconómico

\begin{tabular}{|c|c|c|c|c|c|}
\hline \multirow[b]{2}{*}{$\begin{array}{c}\text { Uso de } \\
\text { Anticoncepcionais }\end{array}$} & \multicolumn{3}{|c|}{ Porto Alegre } & \multicolumn{2}{|c|}{ Cinturão } \\
\hline & $\mathrm{n}$ & $\begin{array}{l}\text { Pressão } \\
\text { Sistólica }\end{array}$ & $\begin{array}{c}\text { Pressão } \\
\text { Diastólica }\end{array}$ & $\mathbf{n}$ & $\begin{array}{l}\text { Pressão } \\
\text { Sistólica }\end{array}$ \\
\hline Nunca & 56 & 120.93 & 70.89 & 81 & 121.46 \\
\hline $\begin{array}{l}\text { Atualmente } \\
<2 \text { anos }\end{array}$ & 15 & 117.67 & 69.87 & 28 & 117.29 \\
\hline$>2$ anos & 75 & 118.27 & 66.53 & 100 & 123.60 \\
\hline $\begin{array}{l}\text { No Passado } \\
<2 \text { anos }\end{array}$ & 43 & 116.12 & 66.83 & 30 & 116.50 \\
\hline$>2$ anos & 65 & 116.46 & 67.03 & 53 & 116.00 \\
\hline
\end{tabular}

TABELA V

Contrastes * das médias de pressão arterial sistólica das usuárias em relação às que nunca tomaram an ticoncepcionais, por estrato geoeconómico

\begin{tabular}{|c|c|c|c|c|c|}
\hline \multirow[b]{2}{*}{$\begin{array}{c}\text { Uso de } \\
\text { Anticoncepcionais }\end{array}$} & \multicolumn{3}{|c|}{ Porto Alegre } & \multicolumn{2}{|c|}{ Cinturão } \\
\hline & Contrastes & E.P. & SIGN. & Contrastes & E.P. \\
\hline $\begin{array}{l}\text { Atualmente } \\
<2 \text { anos }\end{array}$ & 5,20 & 4,53 & NS & 6,00 & 4,07 \\
\hline$>2$ anos & 1,39 & 2,48 & NS & 6,65 & 2,79 \\
\hline $\begin{array}{l}\text { No Passado } \\
<2 \text { anos }\end{array}$ & $-1,61$ & 2,68 & NS & 0,40 & 3,77 \\
\hline$>2$ anos & $-3,13$ & 2,57 & NS & $-0,96$ & 3,11 \\
\hline
\end{tabular}

- Estimativas controladas por idade e índice de Quetelet

\section{TABELA VI}

Contrastes das médias de pressão arterial diastólica das usuárias em relação às que nunca tomaram anticoncepcionais, por estrato geoeconômico

\begin{tabular}{|c|c|c|c|c|c|}
\hline \multirow[b]{2}{*}{$\begin{array}{c}\text { Uso de } \\
\text { Anticoncepcionais }\end{array}$} & \multicolumn{3}{|c|}{ Porto Alegre } & \multicolumn{2}{|c|}{ Cinturão } \\
\hline & Contrastes & E.P. & SIGN. & Contrastes & E.P. \\
\hline $\begin{array}{l}\text { Atualmente } \\
<2 \text { anos }\end{array}$ & 5,00 & 2,89 & NS & $-0,89$ & 2,55 \\
\hline$>2$ anos & $-1,50$ & 1,74 & NS. & 1,56 & 1,71 \\
\hline $\begin{array}{l}\text { No Passado } \\
<2 \text { anos }\end{array}$ & $-0,62$ & 2,00 & NS & $-1,00$ & 2,36 \\
\hline$>2$ anos & $-3,01$ & 1,77 & NS & $-1,94$ & 1,95 \\
\hline
\end{tabular}

- Estimativas controladas por idade e índice de Quetelet 


\begin{tabular}{|c|c|c|c|c|c|c|}
\hline \multirow{2}{*}{$\frac{\text { Metropolitano }}{\text { Pressão }}$} & \multicolumn{3}{|c|}{ Interior Urbano } & \multicolumn{3}{|c|}{ Interior Rural } \\
\hline & $\mathrm{n}$ & $\begin{array}{l}\text { Pressão } \\
\text { Sistólica }\end{array}$ & $\begin{array}{c}\text { Pressão } \\
\text { Diastólica }\end{array}$ & $\mathrm{n}$ & $\begin{array}{l}\text { Pressão } \\
\text { Sistólica }\end{array}$ & $\begin{array}{c}\text { Pressão } \\
\text { Diastólica }\end{array}$ \\
\hline 72.89 & 114 & 117.97 & 66.88 & 135 & 120.30 & 68.69 \\
\hline 65.00 & 27 & 116.52 & 67.25 & 21 & 122.57 & 65.71 \\
\hline 71.28 & 92 & 120.20 & 68.39 & 40 & 122.60 & 68.45 \\
\hline 68.13 & 16 & 115.25 & 67.87 & 14 & 118.86 & 69.86 \\
\hline 67.77 & 31 & 117.16 & 66.19 & 15 & 115.60 & 68.80 \\
\hline
\end{tabular}

\begin{tabular}{|c|c|c|c|c|c|c|}
\hline \multirow{2}{*}{$\frac{\text { fetropolitano }}{\text { SIGN. }}$} & \multicolumn{3}{|c|}{ Interior Urbano } & \multicolumn{3}{|c|}{ Interior Rural } \\
\hline & Contrastes & E.P. & SIGN. & Contrastes & E.P. & SIGN. \\
\hline NS & 2,78 & 3,29 & NS & 6,08 & 3,45 & NS \\
\hline $\mathrm{p}<1 \%$ & 3,32 & 2,06 & NS & 4,55 & 2,63 & NS \\
\hline NS & $-0,90$ & 4,04 & NS & 0,63 & 4,08 & NS \\
\hline NS & $-0,11$ & 2,88 & NS & $-5,31$ & 3,96 & NS \\
\hline
\end{tabular}

\begin{tabular}{|c|c|c|c|c|c|c|}
\hline \multirow{2}{*}{$\frac{\text { Ae tropolitano }}{\text { SIGN. }}$} & \multicolumn{3}{|c|}{ Interior Urbano } & \multicolumn{3}{|c|}{ Interior Rural } \\
\hline & Contrastes & E.P. & SIGN. & Contrastes & E.P. & SIGN \\
\hline NS & 2,96 & 2,32 & NS & $-0,53$ & 2,48 & NS \\
\hline NS & 1,87 & 1,50 & NS & 1,08 & 1,84 & NS \\
\hline NS & 1,88 & 2,83 & NS & 2,87 & 2,89 & NS \\
\hline NS & $-0,89$ & 2,18 & NS & $-1,01$ & 2,81 & NS \\
\hline
\end{tabular}


nível de instrução. Verifica-se que é no grupo das mulheres de escolaridade mais baixa que o efeito dos anticoncepcionais orais é mais acentuado e significativo (Tabela VII).

\section{TABELA VII}

Contrastes* das médias de pressäo arterial sistólica das mulheres residentes na área metropolitana, subdivididas por nivel de instruçāo

\begin{tabular}{|c|c|c|c|c|c|}
\hline Nível de Instrução & $\begin{array}{c}\text { Uso de } \\
\text { Anticoncepcionais }\end{array}$ & $\mathrm{n}$ & Contrastes** & E.P. & SIGN. \\
\hline 1. Primário Incompleto & $\begin{array}{l}\text { Atualmente } \\
\text { No Passado }\end{array}$ & $\begin{array}{l}61 \\
32\end{array}$ & $\begin{array}{r}9,46 \\
-2,85\end{array}$ & $\begin{array}{l}4,54 \\
5,04\end{array}$ & $\begin{array}{l}<1 \% \\
\text { NS }\end{array}$ \\
\hline 2. Primário Comple to & $\begin{array}{l}\text { Atualmente } \\
\text { No Passado }\end{array}$ & $\begin{array}{l}156 \\
159\end{array}$ & $\begin{array}{r}2,53 \\
-0,01\end{array}$ & $\begin{array}{l}1,97 \\
1,93\end{array}$ & $\begin{array}{l}\text { NS } \\
\text { NS }\end{array}$ \\
\hline
\end{tabular}

* Estimativas controladas por idade e indice de Quetelet

* Comparativos com as mulheres que nunca tomaram pílula

Alguns estudos têm discutido o papel da obesidade, fumo e alterações pressóricas na gravidez, associados ao uso de contraceptivos orais, em relação a doenças cardiovasculares.

Comparando-se as médias de pressões arteriais de mulheres fumantes, quanto ao uso de pílulas, através de uma análise de covariâncias, controlando-se idade e indice de Quetelet, não foram detẹctados efeitos significativos. 0 mesmo ocorreu para mulheres obesas.

No entanto, para mulheres com história de hipertensão na gravidez, assim como para aquelas classificadas como hipertensas (critério 140/90 $\mathrm{mm} \mathrm{Hg}$ ), o efeito do uso de pílulas foi bem mais intenso, causando aumentos de mais de $10 \mathrm{~mm}$ de $\mathrm{Hg}$ na pressão sistólica. Esses efeitos foram altamente significativos, com nível descritivo do teste $p$ menor que $1 \%$. (Tabela VIII e IX).

\section{TABELA VIII}

Contrastes * das médias de pressão arterial sistólica das mulheres residentes na área metropolitana, categorizadas segundo alteraçōes *** na gravidez

\begin{tabular}{|c|c|c|c|c|c|}
\hline Alterações na Gravidez & $\begin{array}{c}\text { Uso de } \\
\text { Anticoncepcionais }\end{array}$ & $\mathrm{n}$ & Contrastes ** & E.P. & SIGN. \\
\hline 1. Sim & $\begin{array}{l}\text { Atualmente } \\
\text { No Passado }\end{array}$ & $\begin{array}{l}34 \\
15\end{array}$ & $\begin{array}{r}11,03 \\
4,77\end{array}$ & $\begin{array}{l}4,77 \\
4,82\end{array}$ & $\begin{array}{l}<1 \% \\
\text { NS }\end{array}$ \\
\hline 2. Não & $\begin{array}{l}\text { Atualmente } \\
\text { No Passado }\end{array}$ & $\begin{array}{l}163 \\
144\end{array}$ & $\begin{array}{r}3,52 \\
-1,76\end{array}$ & $\begin{array}{l}2,42 \\
2,40\end{array}$ & $\begin{array}{l}\text { NS } \\
\text { NS }\end{array}$ \\
\hline
\end{tabular}

\footnotetext{
* Estimativas controladas por idade c índice de Quetelet

** Comparativos com as mulheres que nunca tomaram pílula

*** Hipertensão, convulsão, inchaço ou falta de ar
} 


\section{TABELA IX}

Contrastes" das médias de pressão arterial sistólica das mulheres residentes na área metropolitana. categorizadas segundo hipertensão $(140 / 90 \mathrm{~mm} \mathrm{Hg})$

\begin{tabular}{lccccc}
\hline \multirow{2}{*}{ Nível de Condição } & $\begin{array}{c}\text { Uso de } \\
\text { Anticoncepcionais }\end{array}$ & $\mathrm{n}$ & Contrastes** & E.P. & SIGN. \\
& Atualmente & 27 & 13,76 & 5.62 & $<1 \%$ \\
Hipertensas & No Passado & 16 & 7,95 & 6,12 & NS \\
\hline \multirow{2}{*}{ Não-Hipertensas } & Atualmente & 190 & 1,82 & 1,33 & NS \\
& No Passado & 175 & $-1,46$ & 1,32 & NS \\
\hline
\end{tabular}

* Estimativas controladas por idade e índice de Quetelet

** Comparativos com as mulheres que nunca tomaram pílula

\section{DISCUSSÃO}

A alta prevalência do uso de contraceptivos orais no Sul do país foi confirmada nos resultados do nosso estudo, sendo que o uso de pílulas mostrou-se maior nas faixas etárias mais jovens e na área metropolitana.

Essas diferenças por faixa etária parecem refletir uma mudança em atitudes das diversas coortes frente à contracepção, ou seja, mulheres mais jovens fazem maior uso de contraceptivos orais. Este fato é observado em todos os estratos, mesmo no interior rural onde há menor consumo de pílulas. Porém, não se dispõe de informações sobre o uso de métodos alternativos que poderiam explicar, em parte, as diferenças observadas.

Estudos têm mostrado, em geral, que a prática anticoncepcional é mais freqüente em zonas urbanas, em mulheres com nível de instrução mais elevado ${ }^{8}, 9,12$.

$\mathrm{Na}$ nossa pesquisa, foi no Cinturão Metropolitano que se encontrou a maior prevalência do uso de anticoncepcionais orais. Embora não sendo o estrato que apresente o melhor nível de instrução, quando comparado com as outras áreas urbanas, é nele que se encontra a maior proporção de mulheres participantes da força de trabalho no setor terciário de ocupação. Fatores condicionados pelo trabalho, conjuntamente com condições precárias de vida, parecem ter uma influência específica no comportamento dessas mulheres.

Considerando o efeito do uso de pílulas nos níveis tensionais, verificamos que, em todos os estratos, esse efeito foi sempre positivo na média de pressão sistólica. No Cinturão Metropolitano, onde o uso é mais freqüente, esta diferença mostrou-se significativa. Para a pressão diastólica, efeitos na média mostram-se praticamente nulos. Mulheres que deixaram de tomar pílula apresentam, em todos os estratos, pressão sistólica média inferior que nas outras categorias. 
Os resultados encontrados na nossa investigação são consistentes com os de outros autores. A maioria dos estudos realizados para avaliar o efeito dos anticoncepcionais orais na pressão arterial mostra que as mulheres que fazem uso deste método tendem a apresentar, em média, pressão arterial mais elevada. $\mathrm{O}$ efeito mostra-se, em geral, mais significativo na pressão sistólica do que na diastólica ${ }^{2}, 4,6,10,11$.

Há evidências também de que a pressão arterial retorna aos níveis habituais, após interrupção do uso dos anticoncepcionais $^{2}, 11$. Ramcharam ${ }^{6}$ encontrou inclusive níveis pressóricos mais baixos que antes do uso.

Poucos trabalhos investigaram a relação conjunta entre outros fatores de risco e o uso de contraceptivos orais na hipertensão. Clezy et alli ${ }^{2}$ e Saruta et alli ${ }^{10}$ sugeriram um aumento mais significativo na pressão arterial nas mulheres com história prévia de hipertensão na gravidez, enquanto Wallace et alli $^{13}$ constataram alterações positivas na pressão arterial, associadas ao uso combinado de bebidas alcoólicas com anticoncepcionais orais.

A nossa metodologia permitiu avaliar a associação entre o uso de anticoncepcionais orais e outros fatores de risco. Constatou-se que grupos de mulheres com níveis tensionais mais altos na média apresentam um aumento mais nitido na pressão arterial, ao fazerem uso da pîula.

Resultados da Pesquisa Epidemiológica de Hipertensão Arterial no Rio Grande do Sul, publicados anteriormente, mostram que o estrato que apresenta pressão arterial média mais elevada é o Cinturão Metropolitano, para ambos os $\operatorname{sexos}^{3}$. E foi no Cinturão Metropolitano que se encontrou o maior efeito do uso de anticoncepcionais.

Dentre as mulheres residentes na área metropolitana, foi no grupo das mulheres de escolaridade mais baixa, que habitualmente mostram pressão arterial média mais elevada ${ }^{3}$, que o efeito se mostrou mais intenso.

Enfatizamos ainda que, tanto para o grupo de mulheres que apresentavam história de hipertensão na gravidez, como no grupo das mulheres classificadas como hipertensas (critério 140/90), o efeito dos anticoncepcionais orais mostrouse também de grande significância, com alterações de mais de $10 \mathrm{~mm} \mathrm{Hg}$ nas médias de pressão sistólica.

Tais resultados sugerem que os efeitos na pressão arterial causados pelo uso de contraceptivos orais não são homogêneos para todas as mulheres, tanto a nivel individual, como a nivel populacional. Parece que o uso da pílula tende a potencializar o aumento na pressão sistólica em grupos de mulheres já habitualmente com niveis de pressão mais elevados.

Desta forma, deve-se discutir e investigar mais profundamente as implicações do uso indiscriminado da pílula por 
mulheres que apresentam riscos individuais de desenvolver hipertensão, como também por aquelas que fazem parte de um grupo populacional cujo risco devido a características sócio-econômicas é também mais elevado.

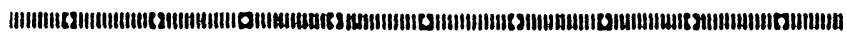

$O$ presente trabalho teve por objetivo descrever a relação entre $o$ uso de anticoncepcionais orais e a pressão arterial em mulheres residentes nas áreas urbana, rural e metropolitana no Rio Grande do Sul, situado no extremo sul do Brasil. Os dados utilizados nesta análise provêm de um inquérito de hipertensão arterial conduzido neste Estado, em 1978.

Através de uma análise de covariâncias, controlando-se idade e indice de Quetelet, as médias de pressão sistólica e diastólica das mulheres usuárias e ex-usuárias de pilulas, foram comparadas com as médias de pressão das mulheres que nunca tomaram anticoncepcionais. Observou-se que, para a pressão sistólica, houve, sistematicamente, nos quatro estratos considerados na pesquisa (Interior Rural, Interior Urbano, Cinturão Metropolitano e Porto Alegre), uma diferença positiva para as mulheres que estão tomando pilula atualmente. Esta diferença mostrou-se significativa $(p<1 \%)$ para as mulheres residentes no Cinturão Metropolitano que tomam anticoncepcionais há mais de dois anos. Para a pressão diastólica, foram observados pequenos efeitos que, embora positivos, não foram significativos.

A metodologia da pesquisa permitiu, ainda, avaliar a associaçāo entre o uso de anticoncepcionais orais e outros fatores de risco. Dentre as mulheres residentes na área metropolitana, foi no grupo das mulheres de escolaridade mais baixa que o efeito se mostrou mais intenso. No grupo de mulheres que apresentavam história de hipertensão na gravidez, assim como nas mulheres classificadas como hipertensas (critério 140/90), o efeito dos anticoncepcionais orais também mostrou-se de grande significância ( $p<1 \%$ ), com alteraçōes de mais de $10 \mathrm{~mm} \mathrm{Hg}$ nas médias de pressão sistólica. Tais resultados sugerem que o uso da pilula tende a potencializar o aumento na pressão sistólica, em grupos de mulheres já habitualmente com niveis de pressão mais elevados.

\section{REFERÊNCIAS BIBLIOGRÁFICAS}

1. BERQUO, E. Algumas indicaçōes sobre a recente queda da fecundidade no Brasil. Teresópolis, 1980. 
mimeo. (notas preparadas para a VI Reunião do Grupo de Trabalho sobre o processo de reprodução da população, da Comissão de População e Desenvolvimento do CLACSO, Teresópolis, abril 1980).

2. CLEZY, T.M. et alii. Oral contraceptives and hypertension; a epidemiological survey. Br. Heart J., 34 :1238.43, 1972.

3. COSTA, E.A. A cross-sectional survey of blood pressure in Rio Grande do Sul, Brasil. London, 1981. (Thesis for the degree of Ph.D., University of Lon. don, School of Hygiene and Tropical Medicine).

4. KUNIN, C.M. et alii. Oral contraceptives and blood pressure. Arch. Intern. Med., $123: 362-5,1969$.

5. MERRICK, T. \& BERQUO, E. The determinants of Brazil's recent rapid decline in fertility. Washington, 1983. mimeo. (Apresentado na National Academy Press, Washington, D.C.).

6. RAMCHARAN, S. et alii. Incidence of hypertension in the walnut creek contraceptive drug study cohort. In: GARATTINI, S. \& BERENDES, H.W. eds. Pharmacology of steroid contraceptive drugs. New York, Raven, 1977. p. 277.88.

7. ROSE, G. Standardization of observers blood pressure measurement. Lancet, $1: 673,1975$.

8. RODRIGUES, W. et alii. Pesquisa sobre saide maternoinfantil e planejamento familiar, Brasil, Região Sul. Rio de Janeiro, BEMFAM, 1982.

9. RODRIGUES, W. et alii. Pesquisa sobre saude maternoinfantil e planejamento familiar, Rio Grande do Norte. Rio de Janeiro, BEMFAM, 1980.

10. SARUTA, T. et alii. A possible mechanism for hypertension induce by oral contraceptives. Arch. Intern. Med., $126: 621.6,1970$.

11. SPELLACY, W.N. \& BIRK, S.A. The effect of intrauterine devices, oral contraceptives, estrogens, and progrestogens on blood pressure. Am. J. Obstet. Ginecol., 112 (7) :912-9, 1972.

12. TAVARES, T. et alii. Prática contraceptiva atual em alguns estados brasileiros. In: ENCONTRO NACIO. NAL ESTUDOS POPULACIONAIS, 3. Vitória, 1982. Anais. Vitória, 1982.

13. WALLACE, R.B. et alii. Alteration in blood pressure associated with combined alcohol and oral contraceptive use. J. Chron. Dis., 35 :251-7, 1982. 
14. WEIR, R.J. et alii. Blood pressure in women taking oral contraceptives. British Med. J., 1 :533-5, 1974.

15. WOOD, J.W. Oral contraceptive and hypertension.

Lancet, 2 :653-9, 1967. 